\title{
Quaderni
}

QUADERNI Communication, technologies, pouvoir

\section{Le Grand Paris qui mange : un projet politique culturel en émergence? [Seconde partie]}

La fabrique symbolique, des processus aux écritures

\section{Dominique Pagès}

\section{(2) OpenEdition \\ Journals}

Édition électronique

URL : http://journals.openedition.org/quaderni/986

DOI : 10.4000 /quaderni.986

ISSN : 2105-2956

Éditeur

Les éditions de la Maison des sciences de l'Homme

Édition imprimée

Date de publication : 5 mai 2016

Pagination : 97-111

\section{Référence électronique}

Dominique Pagès, «Le Grand Paris qui mange : un projet politique culturel en émergence ? [Seconde partie] », Quaderni [En ligne], 90 | Printemps 2016, mis en ligne le 05 mai 2018, consulté le 20 avril 2019. URL : http://journals.openedition.org/quaderni/986 ; DOI : 10.4000/quaderni.986 


\section{Politique}

\section{Le Grand Paris}

qui mange : un

projet politique

culturel en

émergence?

Seconde partie.

La fabrique

symbolique,

des processus

aux écritures

Dominique

Pagès

CELSA, Laboratoire GRIPIC
«Les mots qui vont surgir savent de nous ce que nous ignorons d'eux», René Char.

Le symbolique repose sur un ensemble de représentations que nous nous faisons du monde et par lesquelles nous le comprenons. Ces représentations, plus stables que les simples signes du quotidien, évoluent, se modifient néanmoins, plus ou moins rapidement selon les époques, témoignant des transformations de nos sociétés et appelant à des interprétations en continu. Le symbolique ouvre donc aux relations entre les symboles qui circulent pour permettre que le collectif (à des échelles multiples) se fonde autour de valeurs potentiellement communes. Le symbolique désigne non seulement les signes symboles mais aussi des rituels, des cérémonies, des pratiques (tels les actes de fondation ou de refondation) qui traduisent, transmettent et actualisent (de manière plus ou moins durable, 'efficiente' et acceptée) ces valeurs. La « fabrique » symbolique, quant à elle, désigne un mode d'action : elle constitue un travail de sélection et d'agencements de signes et de symboles, de formats et de supports, tente d'ordonner des pratiques. Dans la perspective de l'avènement du Grand Paris, ce travail symbolique ne fait donc pas qu'accompagner le processus de métropolisation mais le fonde, l'incarne, le rend perceptible et, possiblement, appropriable ${ }^{1}$. Ainsi, cherchet-il à permettre aux habitants métropolitains de faire évoluer leur cadre de référence et leurs interprétations mais aussi à les faire progressivement entrer dans un nouvel ordre urbain, et notamment celui d'une métropole dite durable et résiliente où la nature retrouverait pleinement sa place, ses rythmes, ses logiques (dites « éco-systémiques »). 
À la lumière de ces premières précisions, les lieux agri-urbains envisagés dans le précédent article ne visent donc pas qu'à subvenir aux besoins alimentaires : ce sont aussi des lieux de culture dans tous les sens du terme, ceux d'une remise en cause de nos modèles de développement, de nos actuelles représentations de la nature, de la terre et de la ville; ce sont aussi des lieux de conscientisation où une science de la nature se partage et s'enrichit au fil d'apprentissages divers. La stratégie alimentaire métropolitaine ne recouvre pas que des objectifs fonctionnels, écologiques, environnementaux, économiques ou technologiques : ses enjeux sont aussi symboliques, culturels et anthropologiques. Les mutations agri-urbaines invitent tant à une reconquête culturelle de nos assiettes, à la redécouverte du goût qu'à celle, patrimoniale, de nos produits, tout en participant au vivre ensemble, au lien commun, au questionnement de nos cultures alimentaires et culinaires (et à leur influence sur les crises actuelles).

Ce passage du projet environnemental au projet culturel s'accomplit notamment au fil de différents processus qui cherchent à donner un sens commun à ces mutations. Nous évoquerons ainsi trois d'entre eux (l'évènementialisation, la patrimonialisation, la mise en tourisme) avant de questionner plus précisément les formes de récits médiatiques et artistiques qui participent à la démocratisation de la métropole nourricière.

\section{Les processus culturels de développement de «la Métropole qui mange»}

\section{«Les mots justes trouvés au bon moment sont de} l'action », Hannah Arendt.
Le travail symbolique (notamment institutionnel) qui accompagne la mise à l'agenda (politique, médiatique, scientifique...) des villes nourricières, et plus précisément de la reconfiguration de nos systèmes alimentaires, ouvre à la production de signes mais aussi à des actes impliquant les habitants et les visiteurs. Ainsi, des évènements culturels et, progressivement, des débats, des consultations s'organisent cherchant à relier les acteurs en présence et à favoriser l'intégration active au projet d'une métropole dite fertile, nourricière ou comestible.

\section{L'évènementialisation}

Ainsi, la fabrique symbolique du Grand Paris passe par la fête et, plus largement, par les évènements culturels festifs dont le pouvoir de rassemblement et la capacité de mise en réseau des territoires locaux constituent une réserve de significations dynamiques. L'évènement culturel, qui devient l'un des moyens de communication territoriale privilégié de ce début du XXI ${ }^{e}$ siècle, est à la fois un temps, une forme et un espace symboliques, producteurs de sens. Il mobilise autour d'une nouvelle territorialisation, et, en tant que cérémonie démocratique contemporaine, permet à la métropole de se signifier collectivement, de se rendre visible et perceptible, de susciter du lien dans des sociétés caractérisées par une certaine fragmentation et diverses formes de solitude. En mettant en réseau des territoires centraux et périphériques, l'évènement culturel métropolitain signifie et met en intrigue la métropole, l'inscrit dans 'l'horizon d'attente' et la mémoire tant de ceux qui l'habitent que de ceux qui la visitent ${ }^{2}$.

Ces évènements (plus ou moins conceptuels, 
esthétiques, participatifs) sont envisageables culturellement mais aussi de manière socialement 'performative' : non seulement ils participent à la réécriture des rythmes de la métropole (via son agenda culturel) mais ils transforment (certes de manière éphémère), lieux et espaces, promettant de les requalifier voire de les régénérer ${ }^{3}$. En désignant et en qualifiant le territoire métropolitain, ils y attachent des connotations positives et patrimoniales, festives et ludiques. Par la 'mise en évènement' de la question alimentaire et agriurbaine, il s'agit donc d'adosser une dimension monstrative et festive aux métropoles nourricières - et ceci pour en signifier le sens, en publiciser les enjeux et en vulgariser les attributs, pour faire prendre la mesure des changements en cours. L'évènement attire l'attention publique sur une vision 'autre', appelle à un changement d'attitude, participe à l'interpellation des habitants et les invite à questionner les projets agri-urbains en cours et à s'engager. Nous évoquerons quatre récents évènements emblématiques de la diversité des écritures festives de la métropole nourricière.

Ville fertile (www.citechaillot.fr), l'invitation utopique : cette exposition plurielle montée à la Cité de l'Architecture et du Patrimoine (en 2011) actualisa les représentations utopiques de la ville féconde et du « jardin planétaire » (même si les thématiques de l'agriculture urbaine et de la ville nourricière n'étaient que fort marginales). Sa dimension immersive, la puissance évocatrice de sa scénographie remportèrent l'adhésion de publics venus en nombre. Par la richesse des interviews (experts et grands témoins) et celui des supports éditoriaux déployés, par la dimension comparative de son parcours (les métropoles mondiales innovantes), cet évènement permit aussi d'enrichir les débats sur la nature urbaine, ici et ailleurs. Cet évènement jouait sur les esthétiques de la refondation, tout à la fois celle de la ville et de la nature.

Ma Cantine en Ville (www.citechaillot.fr), une approche communautaire : coproduite en 2013 par le VIA et la CAP, cet évènement (présentant les résultats du concours de micro-architecture Minimousses) rassembla professionnels et amateurs, étudiants et chercheurs, associations et entreprises. Décloisonnant ainsi les pratiques et les métiers, l'exposition favorisait un questionnement transversal sur la production et la consommation alimentaires urbaines, elle se souciait de croiser les réseaux existants et d'inclure des acteurs a priori éloignés du sujet mais porteurs de projets innovants, répondant aux questions conductrices de l'évènement. Comment nourrir et se nourrir dans la densité et l'extension infinie de nos villes? Comment faire pour que ces moments soient facteurs de sociabilité et d'innovation sociale?

Carrot City (www.carrotcity.com), un évènement dialogique: cette exposition itinérante (créée en 2009 à l'Université Ryenson de Toronto et qui a pris place au Parc de Bercy à l'été 2013, après être passée par New York et Berlin) portait plus explicitement sur les projets novateurs en matière de production alimentaire durable en ville, explorant les liens renouvelés entre la ville et l'agriculture, invitant à réfléchir à l'agri-design et aux conditions d'une mobilisation citoyenne. Au fil de ses « voyages », elle s'est enrichie d'expériences locales sous forme de photographies et de visuels, illustrant la conception, l'aménagement et le développement des projets d'agriculture 
urbaine et nourrissant la réflexion sur la revitalisation des différents espaces urbains, centraux ou périphériques.

Savez vous planter les choux au Parc de Bagatelle, une pédagogie bon enfant : s'interroger sur l'alimentation en ville et, plus spécifiquement, 'faire découvrir l'histoire du maraîchage en Île-de-France' mais aussi 'fêter l'agriculture urbaine et périurbaine' furent les enjeux de cet évènement, en 2013. Producteurs des environs de Paris, acteurs des marchés, des halles mais aussi légumes oubliés ont été à l'honneur dans les bâtiments et dans le parc. Enfants et adultes ont été invités à découvrir produits locaux (légumes, fruits, plantations diverses), à découvrir de nouvelles façons de nourrir Paris demain, revisitant ainsi les valeurs de leur appartenance territoriale et réfléchissant aux formes inédites d'un retour à la terre.

Le cycle Cultiver Partout à Paris organisé par la Mairie de Paris (8 conférences thématiques organisées de mai 2015 à janvier 2016) : le succès de cet évènement sembla surprendre même les organisateurs tant les publics (de tous les âges, de tous les statuts sociaux) furent massivement au rendez-vous dans une diversité de salles municipales et publiques, tant le relais des réseaux sociaux fut actif et s'amplifia. Ce cycle a permis d'envisager la diversité des compétences en jeu, des terrains d'expérimentation, des initiatives métropolitaines en présence. Des débats se sont amorcés sur des questions concrètes (les conditions du devenir agriculteur urbain, la fiscalité de l'agriculture urbaine, la diversité des approches de permaculture, les start ups dédiées à l'agriculture urbaine, etc.). Les dialogues entre scientifiques, militants, porteurs de projets et citoyens furent intenses et annonciateurs d'autres évènements pour 2016-2017.

L'évènementialisation de la ville alimentaire, culinaire et gourmande (dont les énonciateurs et les registres discursifs sont donc multiples), ce sont aussi ces déclinaisons parisiennes d'évènements internationaux que certains chercheurs analysent comme des formes d'urbanisme temporaire, d'aménagement du temps urbain : la Fête de la gastronomie (www.economie.gouv. fr), Taste of Paris (www.tasteofparis.fr) en sont emblématiques ; c'est aussi une diversification croissante d'évènements plus confidentiels ${ }^{4}$ : tel le festival Éclosion urbaine ${ }^{5}$ porté par la SAUGE (Société d'Agriculture Urbaine, Généreuse et Engagée qui lance en 2016 une nouvelle initiative, Adopte une graine - www.adopteunegraine. com), le Salon des Plaisirs gourmands, le Gourmet food truck festival, ou encore les Marchés flottants. Ces évènements, aussi contrastés soient-ils dans leur positionnement et dans leur rhétorique, cherchent à signifier un tournant, le passage vers une nouvelle ère, à inciter à d'autres façons de penser et de faire. Ils mettent en scène une réconciliation avec la nature et se construisent comme des réponses : leur visée est réparatrice mais aussi anticipatrice, nostalgique mais aussi prospective. Bien sûr leur réception serait à questionner (chacun a sa genèse propre, sa scénographie, ses conditions de structuration) : comment ces propositions culturelles sont elles qualifiées par les publics ? Comment s'intègrent-elles dans le calendrier festif du Grand Paris ?... Mais il n'est pas lieu ici de répondre à ces questions, appelant de fines études de terrain. 


\section{La patrimonialisation}

La production symbolique d'un territoire participe d'un projet de transmission. Reliant passé, présent et futur, le processus de patrimonialisation du Grand Paris s'amorce depuis quelques années à peine et redessine notamment (en tant que processus symbolique d'apparition, de légitimation et de partage métropolitains) le rapport de Paris à la nature, aux terroirs et à la mémoire culinaire, à la production alimentaire. Illustration de cette tendance, la valorisation patrimoniale des produits locaux franciliens et métropolitains, redécouverts ou réinventés : elle se consolide via une diversité d'intermédiaires et d'intermédiations (acteurs du patrimoine, artisans de bouche, sites internet divers), soucieuse de la renaissance de légumes, de la redécouverte de plantes, de semences oubliées ou reléguées. Elle se professionnalise, diversifiant les signes de qualité et précisant de plus en plus finement ces indications géographiques : des actions d'inventaire des patrimoines alimentaires locaux sont en cours; des produits de terroir, des fabrications artisanales et des spécialités culinaires trouvent place dans des boutiques dédiées ; des dégustations de produits locaux se multiplient notamment à l'occasion des évènements évoqués précédemment pour faire reconnaître la richesse des patrimoines alimentaires et culinaires locaux.

Un organisme, unique dans son genre, accompagne cette dynamique de professionnalisation : le CERVIA, chargé tant de la valorisation et de l'innovation agricole et alimentaire en Île-deFrance que du soutien et du développement économique des entreprises agricoles et alimentaires franciliennes. En pointe sur les problématiques liées à l'alimentation locale, aux circuits courts et à la diversification agricole, le CERVIA regroupe à la fois les missions d'un Comité de promotion des produits agricoles et alimentaires et celles d'un Centre Régional d'Innovation et de transfert de technologies (CRITT). La marque Saveurs Île-de-France (www.saveursparisidf.com) qu'il a lancée en 2011 participe à cette émergence de nouveaux produits de terroir - envisagés comme une richesse patrimoniale et un potentiel de développement ainsi qu'à la redécouverte de produits porteurs de mémoires culinaires : elle vise à identifier les produits alimentaires cultivés ou élaborés en Île-de-France et à donner une visibilité aux producteurs et artisans 'remarquables' du territoire et à dynamiser l'économie locale. Son logo se décline en trois couleurs : vert pour les produits agricoles cultivés ou produits en Île-deFrance, bleu azur pour les produits fabriqués par les artisans des métiers de bouche, rouge et gris pour les produits de petites et moyennes entreprises de transformation agro-alimentaire. Cette marque, qui veut ainsi exprimer une 'identité' alimentaire fédératrice, fonctionne comme un signe de ralliement et de reconnaissance pour toute la filière. Son approche est globalisante : il s'agit tant d'identifier à l'export les produits franciliens et métropolitains que d'apporter une aide au consommateur (apprendre à mieux choisir) que de toucher les 40 millions de touristes en visite chaque année. Dans ce sens, le CERVIA développe d'autres actions grand public : une démarche collective Mangeons local en Île-deFrance qui est basée sur une activité ayant trait à l'environnement, le patrimoine et l'aspect social et a pour objectif de soutenir et d'accompagner l'ensemble des acteurs de la filière agroalimentaire Du champ à l'assiette de la région capitale ; 
un label, Des produits d'ici, cuisinés ici (2013) pour valoriser les chefs restaurateurs franciliens, 'Le label des chefs franciliens qui cuisinent local'.

Ces différentes réécritures patrimoniales étoffent le répertoire culinaire du Grand Paris, le documentent et font de la gastronomie une thématique en puissance pour sa stratégie de développement. Il ne s'agit pas seulement de proposer des produits sains mais de redonner au territoire productif une valeur mémorielle forte et aux habitants la connaissance des terroirs, le goût des bons produits et du cuisiner local.

\section{La mise en tourisme}

La fabrique métropolitaine, c'est donc ce travail de production de références symboliques qui peut prendre de multiples formes (l'évènementialisation, la patrimonialisation mais aussi, celles, complémentaires, de la mise en tourisme et de la narration dans son sens le plus créatif qui soit). Via le tourisme et notamment le tourisme dit métropolitain, la gastronomie devient pour le Grand Paris une activité économique, créative et symbolique stratégique. Il s'agit de proposer aux touristes des lieux inédits, de nouveaux produits locaux, des saveurs renouvelées et donc de faire évoluer la perception élitiste de la gastronomie parisienne. Ainsi diverses récentes initiatives des Comités Départementaux du Tourisme du Grand Paris $(92,93,94)$ témoignent d'une volonté de remettre en question les hiérarchies alimentaires et culinaires en place (entre les territoires). Leurs sites internet, qui intègrent une entrée ou une rubrique " gastronomie », peuvent ainsi proposer une cartographie des restaurants $d u$ territoire (invisibles dans les guides touristiques), inviter à des promenades agritouristiques et gourmandes (certes à documenter et publiciser plus avant), faire connaître des traditions culinaires oubliées.

Désigné par certains comme une sorte de Beaubourg de la gastronomie, le projet de la Cité de la Gastronomie de Rungis (s'inscrivant, avec Tours, Lyon et Dijon, dans un réseau) devrait, s'il est réalisé, en faire un lieu phare de ce tourisme à la fois agro-alimentaire et culinaire, gourmand et gastronomique (chaque appellation recouvrant tant un imaginaire des métiers, une offre que des attentes spécifiques) : envisagée à la fois comme lieu d'expositions et de recherches, de rencontres mais aussi espace didactique de découverte et de dégustations, cette Cité, centrée sur la reconquête du goût et sur la créativité de la gastronomie francilienne et métropolitaine, devrait donner au " Grand Paris qui mange » une envergure à la fois culturelle, touristique et économique inédite.

La thématique gastronomique peut ainsi s'articuler à la thématique alimentaire en recombinant les patrimoines et les lieux existant, en organisant le territoire métropolitain autour d'un thème rassembleur, évocateur, cohérent et partagé. Sur le terrain, jardins, fermes, potagers urbains se relient ainsi de plus en plus aux restaurants, aux boutiques labellisées, aux ateliers de dégustation et aux autres lieux culturels que sont notamment les musées, les théâtres, les centres d'art. Cette thématique croisée (alimentaire, culinaire, gastronomique) apparaît mobilisatrice, inscrite dans le projet d'une métropole durable et ouverte à des énonciations renouvelées. 
Les écritures médiatiques et artistiques du Grand Paris qui mange

«Les mots disent le monde et les mots disent l'homme », Paul Eluard.

La métropole qui mange c'est, en effet, aussi celle qui le dit et se le dit : divers récits ont été abordés (l'institutionnel de manière privilégiée), d'autres restent à évoquer. Actualisant, traduisant et accompagnant les processus envisagés, diverses productions éditoriales (guides, sites internet, blogs, documentaires, performances artistiques) construisent, chacune à leur façon, un regard renouvelé sur les métropoles en transition alimentaire et notamment celle du Grand Paris.

\section{Écritures et lectures d'un territoire agri-urbain}

Cette problématique de la métropole en transition alimentaire est investie par des acteurs, des collectifs aux approches et aux intérêts contrastés, si bien que celle-ci s'inscrit progressivement dans nos quotidiens par des mots et des images de plus en plus diversifiés, au fil de représentations qui circulent, s'échangent, se discutent pour rendre compréhensibles et tangibles les mutations en cours. Par cette activité narrative plurielle, elle nous devient familière : le " Grand Paris qui mange ", c'est cet ensemble d'enjeux, d'initiatives, d'acteurs mais avant tout de discours, plus ou moins lisibles et audibles, qui participent à façonner une vision composite, susceptible de rallier un nombre important d'habitants, de porteurs de projets, d'organisations. Il s'agit d'expliquer l'émergence d'un nouveau modèle, de le mettre en débat, de donner la parole à une diversité d'énonciateurs et d'inviter les citoyens à s'y impliquer d'une manière ou d'une autre. Il s'agit de trouver progressivement un langage commun à l'ensemble des parties prenantes et, plus largement, de « mettre en désir » un Grand Paris nourricier, de le documenter, de laisser une activité narrative se déployer autour de lui, d'illustrer ses valeurs environnementales, sociales et culturelles : cela passe donc par une diversité d'énonciateurs, mais aussi de médias et de supports, et donc par une pluralité d'écritures.

Cet article et celui qui l'a précédé témoignent de cette diversité des écritures, actualisant la diversité des points de vue et des stratégies en place. Ainsi, à ce point de notre avancée, peut-on identifier six registres de discours, non exclusifs, énonçant le " Grand Paris qui mange », à savoir les discours scientifique, public, urbanistique, culturel et touristique, associatif, artistique. Cette première catégorisation se déduit d'une simple consultation sur Google (le plus fréquenté des moteurs de recherche) à partir d'une série d'associations de mots : «métropole agricole », «métropole agri-urbaine », «métropole/ville fertile», «métropole/ville nourricière », «métropole/ville comestible », « métropole/ville vivrière », « métropole/ville gourmande ». Apparaissent (dans les deux premières pages) les acteurs les mieux référencés sur ces questions, et notamment des métropoles et des territoires mais dans un ordre d'apparition singulièrement dispersé. Ainsi pour l'entrée «métropole agricole » : c'est le Grand Paris qui prédomine; pour l'entrée «métropole agri-urbaine» : la vallée du Rhône, Bordeaux et Rennes ; pour « métropole/ville fertile » (en termes alimentaires) : Nantes, Metz, Montpellier ; pour «métropole/ville nourricière »: Québec et Sherbrooke mais aussi Strasbourg et Nantes ; 
pour «métropole/ville comestible » : Nantes, Arcueil, Limoges, Orléans; pour « métropole/ ville vivrière » : Nantes, Rennes, Limoges; pour « métropole/ville gourmande » : Rennes, Metz, Orléans, Montpellier, Saint-Étienne.

Si nous poursuivons nos investigations en allant directement vers les sites d'acteurs fréquemment évoqués comme exemplaires ou représentatifs des villes en transition alimentaire (notamment lors des évènements organisés par la Ville de Paris), nous retrouvons les six registres discursifs préalablement évoqués (que nous ne ferons ici qu'envisager, d'autres études devraient les investir de manière plus systématique) :

- Au titre des discours scientifiques qui invitent à un Grand Paris de l'expérimentation, des projets résilients (des agronomes aux géologues, des paysagistes aux politistes), ce sont les CAUE d'Île-de-France, l'AIGP (Atelier International du Grand Paris), l'École AgroParistech mais aussi le Muséum d'Histoire Naturelle qui se distinguent. Leur vocation est de construire et de diffuser des savoirs objectifs qui sont 'traduits' et relayés par des vulgarisateurs en nombre croissant (pour exemple l'association Vergers Urbains - vers une ville Comestible, créée en $2012^{6}$ - www.ville. comestible.org).

- Au titre de la communication publique institutionnelle (déjà envisagée), la Mairie de Paris joue un rôle de proposition et d'entraînement, cherchant à sensibiliser à un modèle de ville en transition agri-urbain et à éduquer aux mutations du système alimentaire, invitant à de nouveaux modes d'action, à des engagements précis et quotidiens. Ainsi en est-il de Paris Habitat qui a démultiplié les expérimentations en termes d'agriculture urbaine dans son parc locatif, du petit potager au parc agri-urbain. En valorisant une diversité d'expériences associatives et citoyennes, la voix de Paris semble actuellement incontournable pour restaurer localement la confiance en l'alimentation, pour faire face à la publicité des industries alimentaires mondiales (en contestant sur le terrain public la communication des marques) mais aussi pour faire comprendre la fin proche d'une abondance alimentaire,

- Pour ce qui concerne les acteurs marchands, la plupart des sites en présence valorisent surtout une métropole alimentaire et gourmande 'talentueuse', 'innovante', celle des 'arts de vivre'. Mais de nouveaux acteurs apparaissent, notamment via Internet (blogs, plateformes, applications) : ainsi Alimentation générale (www.alimentationgenerale.net $)^{7}$, une start up parisienne au projet transversal qui édite un magazine indépendant et participatif (traitant de l'ensemble des sujets politiques, sociologiques, économiques et culturels liés à l'alimentation, du champ à l'assiette), un guide des acteurs du « bien manger» dans le Grand Paris et en France (respectant la biodiversité, le plaisir du goût et du savoir faire) qui propose des activités d'aide aux initiatives et porteurs de projets « alimentation » mais aussi qui conçoit des objets éditoriaux et des éditions spéciales, des publicités et développe des partenariats. L'innovation éditoriale (tant en termes de management que d'écriture) semble être au rendez-vous des mutations alimentaires urbaines et agri-urbaines. Citons aussi Urbagri (www.urbagri.org), conseil auprès des porteurs de projets agri-urbain, Agricool (www.agricool.com) qui produit des fruits dans un container localisé dans le Parc de Bercy 
(une expérimentation privée accompagnée par la Mairie de Paris et dont le projet est métropolitain) ou encore, en périphérie, Plaine de Saveurs, un centre de ressources multi-pôles.

- Les discours touristiques sont sans doute les plus contrastés : nombre de guides gastronomiques parisiens, édités localement, valorisent encore un modèle gastronomique (avec ses lieux, ses rites et codes) relativement classique, ainsi, Paris Resto et Paris Gourmand du Petit Futé, 2014 ou Paris Gourmand, un des cities guides distribués par l'OTCP); des guides édités à l'étranger (anglo-saxons) s'efforcent de découvrir de nouvelles adresses et de valoriser de nouvelles expériences gourmandes mais n'en n'oublient pas moins majoritairement les périphéries (ainsi, The Food Lovers guide to Paris, de Patricia Wels, le Good Food in Paris original et le Paris Gourmet Food@guide, Paris Insider guide, édités entre 2011 et 2014 ). Depuis peu des 'éco-guides', des guides pour 'locavores' (ceux qui ne s'alimentent qu'avec des aliments produits dans un périmètre de 150 à $200 \mathrm{kms}$ maximum), plus curieux des marges et des périphéries invitent le visiteur à la découverte d'un Grand Paris locavore, cherchent à donner aux habitants et aux visiteurs les clés pour aller découvrir les producteurs locaux. Ainsi : le Guide du Locavore de Anne Sophie $\mathrm{Novel}^{8}$ qui invite à 'renouer avec la terre grâce à l'alimentation saine et locale', à 'remettre l'humain et l'environnement au cœur des habitudes de consommation', à modifier son alimentation en devenant locavore ; le Guide Paris Ville Comestible, guide de l'Agriculture urbaine en 100 lieux de Gaëtan Laot $^{9}$ qui répertorie vignes, ruches, potagers, rappelant que la capitale et sa proche couronne regroupent de petites exploitations agricoles, tout en mettant en avant le patrimoine passé. Ces guides sont autant d'invitations à mieux connaître les produits locaux soucieux de la biodiversité et de la transition écologique.

- Quatrième discours, celui des architectes et des urbanistes (cherchant à intégrer l'agriculture dans l'aménagement de la ville). Celui-ci est à la fois pluriel et personnalisé, produit par des innovateurs plus ou moins médiatisés (Vincent Callebaut, Constantin Petcou (précédent article), d'autres souvent liés à des territoires précis). Oscillant entre témoignages d'expériences réalisées $(R$-urban) et approches visionnaires ou prospectives (Urbanscrapers agricoles), la gamme des possibles s'enrichit depuis quelques années, notamment en réponse à des appels à initiatives publics.

- Les écritures associatives et militantes d'une métropole «qui mange » les plus visibles sur internet sont avant tout celles de porteurs de projets bien identifiés, animant souvent des lieux dits alternatifs - notamment répertoriés par les guides locavores, par la Mairie de Paris, par des médias culturels. Ces acteurs qui se décrivent souvent comme des lanceurs d'alerte sont issus de mouvements militants divers mais chacun valorise une métropole agri-urbaine et/ou nourricière où l'on s'engage, partage, met en commun : il en est ainsi pour V'île Fertile ${ }^{10}$ qui veut devenir un refuge exemplaire de la biodiversité ; pour $L a$ Recyclerie ${ }^{11}$ qui articule une diversité d'espaces et dont les choix graphiques très créatifs témoignent d'un projet tant écologique que culturel. D'autres déploient des discours plus offensifs comme celui Terres Fertiles (qui dénonce les spéculations immobilières liées au Grand Paris, dévoreuses 
d'espaces agricoles) ou plus personnels comme Paris paysanne (un blog dont l'auteur américaine veut sensibiliser les consommateurs à l'importance de soutenir les productions en achetant des produits locaux).

- Les écritures artistiques, quant à elles, questionnent cette métropole qui mange et se mange avec gravité ou poésie, simple malice ou humour décalé, pessimisme ou optimisme. L'alimentation et ses produits, ses rites et ses scènes font partie de notre histoire de l'art (de la scène de festin à la nature morte, du repas champêtre à la boîte de conserve industrielle). Mais ce début de siècle semble marquer un renouvellement politique des regards. Depuis quelques années, des artistes interrogent de multiples manières notre relation à la ville alimentaire, les rituels culinaires et gourmands qui s'y déploient, éprouvant en termes esthétiques, critiques et/ou ludiques ce modèle de la métropole comestible et fertile.

Nous n'évoquerons ici que trois approches artistiques, choisies pour leur spécificité formelle :

- celui d'une photographe (Mathilde de l'Écotais qui travaille la matière alimentaire pour créer des paysages urbains ou planétaires singuliers) et notamment l'œuvre New York concombre, tissée à partir d'épluchures et de chair de concombre (www.mathilidedelecotais.com) ${ }^{12}$;

- celui d'un collectif d'artivistes, Les Saphrophites, regroupant architectes, scénographes et plasticiens (www.les-saprophytes.org). Fervents défenseurs du recyclage, ils se regroupent autour de projets communs liés à une réflexion sur la place de l'homme dans l'espace public et dans son milieu. Ils installent de singulières cuisines urbaines éphémères, invitent à des expériences alimentaires et à de nouveaux rituels urbains (fondés sur les ressources locales et jouant sur nos peurs et désirs alimentaires), proposent des performances autour de l'agriculture urbaine. Ils mettent notamment en place des processus de construction collective de la ville, de microurbanisme alimentaire basés sur la rencontre alimentaire, l'échange de pratiques culinaires et de savoir-faire (plantations d'arbres fruitiers urbains, création de singuliers potagers éphémères, organisation de festins de quartier, co-rédaction de guides subjectifs de quartiers, projet de ferme urbaine - et ceci avec le soutien ponctuel de l'Institut Français) ;

- enfin celui d'une documentariste, Marie Monique Robin qui a réalisé en 2014, avec le soutien d'Arte, Sacrée Croissance - film accompagné d'une publication où elle évoque via la voix de chercheurs et d'habitants engagés la diversité des luttes pour la préservation de la biodiversité, pour le commerce équilibré, pour les circuits courts, pour la redécouverte des traditions agricoles.

Le documentaire indépendant semble s'affirmer, depuis quelques années dans ce questionnement des villes nourricières et de leurs imaginaires, des crises alimentaires mais aussi d'expériences alternatives emblématiques : de Coline Serreau (Solutions locales pour un désordre global, 2011) à Erwan Wagenhoofer (We feed the world, le marché de la faim, 2012) ou encore Jean-Paul Jaud (Tous cobayes, 2012) et Robert Kenner (Food Inc - les alimenteurs au Québec, 2012), le documentaire témoigne, plus que tout autre média artistique, des contestations, des conflits 
autour des industries mais aussi des politiques alimentaires. Ce genre filmique, avant tout militant, permet en effet de rendre compte de la profusion, à travers le monde, des collectifs dénonçant les risques liés au choix alimentaires des villes. L'écriture documentaire éprouve les discours des industries agroalimentaires, valorise des modes de production et de distribution alternatifs, défend une consommation critique. Elle peut ainsi tout à la fois révéler les dérives et coulisses de l'industrialisation alimentaire, relier et analyser des expérimentations locales engagées (ainsi au niveau de métropoles comme Toronto, Rosario, Montréal ou Vancouver, Détroit ou encore Amsterdam) et ouvrir le débat à une échelle internationale. On peut remarquer un récent glissement de ton dans ces films : de dramatique et sombre, celui-ci semble se vouloir porteur d'espérance collective (ainsi le Demain de Cyril Dion et Mélanie Laurent, 2015). Pour autant, ces films ne se limitent pas au seul souci de faire état du réel : entre pamphlet et ode, invitation à changer le monde et à le 'désaliéner du capitalisme', ils veulent sensibiliser voire choquer, éveiller et faire réagir, tout en exaltant le monde du vivant.

Autant de discours donc qui cherchent à faire rêver ou comprendre, à séduire ou convaincre, à alarmer ou à galvaniser : cette diversité des régimes discursifs est à prendre en compte pour éviter la simple déclinaison d'un modèle univoque et standardisé de la «métropole nourricière » sur notre territoire, pour ne pas la limiter aux seuls enjeux environnementaux. Pour qu'une économie symbolique fonctionne, il faut bien sûr qu'elle rencontre l'adhésion, la confiance, l'appropriation de son public ou plutôt de ses publics, car les populations de la métropole sont d'origines (sociales, ethniques, culturelles, religieuses) très variées. Longtemps les habitants ont été tenus à l'écart pour que le projet du Grand Paris se discute et s'affirme politiquement, pour qu'il se partage tout d'abord entre élus, experts et observateurs : mais ils sont et seront de plus en plus appelés à s'exprimer, soit de manière sélective et thématisée (sur une dimension de l'agriculture urbaine), soit d'une manière plus large et ouverte (sur le projet global de métropole nourricière). Ainsi, les dispositifs de participation démocratique (physique et numérique) s'affinent pour que les métropolitains prennent la parole, énoncent leur points de vue, fassent part de leurs représentations, expriment leurs oppositions voire contrepropositions. Mais pour que les citoyens prennent position en pleine conscience, la qualité de leur information et celle des processus de la communication citoyenne (et plus génériquement de la diffusion des savoirs autour des questions agri-urbaines et alimentaires) sont tout à la fois des préalables et des aboutissements.

Gouverner « la métropole qui mange » devrait ainsi renvoyer moins à l'imposition de décisions venues d'en haut, au nom de l'intérêt général, qu'à la mobilisation, l'argumentation, la négociation pour élaborer en commun des consensus autour de la transition alimentaire, en prenant en compte les divergences des approches. Partant de l'hypothèse que ce seront de plus en plus ces individus, leurs comportements et leurs interactions qui font et feront évoluer la métropole nourricière, il est nécessaire d'envisager plus finement leurs expériences alimentaires et culinaires quotidiennes en tant qu'ensemble de manières de vivre et de penser la métropole. Dans cette perspective, on ne peut que souligner l'importance 
de cette profusion croissante de textes, de récits, de discours permettant de sortir d'une approche trop verticale de la gouvernance. L'expression de tout un chacun sur notre devenir urbain alimentaire, la confrontation des points de vue sur une métropole entre « agriculture hightech et paniers de légumes » participent de plus en plus d'une métropole dite 'durable'.

Ainsi la «métropole qui mange » se fonde sur une production de symboles vivants, appelant des interprétations dynamiques et plurielles. Questionner plus finement l'efficacité de ces signes et de ces symboles qui circulent (fondent-ils des accords, des appropriations, des échanges ?) nous invite à des études sémiologiques et anthropologiques à venir

\section{Conclusion}

Le symbolique est au cœur de la fondation métropolitaine : il l'incarne, donne chair à la métropole, la rend tangible, substantielle pour que celle-ci ne se résume pas en un simple lieu de circulation, d'aménagement ou de résidence mais devienne un lieu d'expression, de significations et de sens. Permettre l'avènement de la métropole qui mange, c'est donc changer le regard sur des territoires et interroger les évolutions de notre environnement alimentaire et culinaire. C'est donner à ce projet alimentaire métropolitain une dimension signifiante pour tous, aussi contradictoire ou hétérogène soit-elle, permettre que cela fasse sens malgré les dissensions.

Ainsi, penser le Grand Paris qui mange c'est ne pas se contenter de l'approche économiste (centrée sur les questions d'approvisionnement des villes) ou écologiste (centrée sur les questions politiques et culturelles de préservation, de conservation, de sauvegarde). C'est penser les mutations culturelles à l'œuvre en termes de représentations, de croyances, de modes de vie, de consommation dans un sens symbolique (nouvelles valeurs, nouveaux rituels, etc.). Ni les professionnels de l'agriculture, ni les industriels agro-alimentaires, ni les institutionnels du développement, ni la société civile, ni les chercheurs disciplinaires ni les praticiens généralistes ne sont la clé du problème. C'est ensemble, en chaque lieu, à chaque instant que s'énonce le problème de 'la métropole qui mange' et s'ébauche si ce n'est la solution du moins un horizon plus lisible. 


\section{$R \cdot E ́ F \cdot E \cdot R \cdot E \cdot N \cdot C \cdot E \cdot S$}

Violaine APPEL, Cécile BANDO, Hélène BOULANGER, Gaëlle CRENN, Valérie CROISSANT, Bénédicte TOULLEC, La mise en culture des territoires, nouvelles formes de culture évènementielle et initiatives des collectivités locales, Nancy, Presses Universitaires, 2008. Lionel ASTRUC et Cécile GROS, Manger local, Actes Sud, Collection Domaine du possible, 2011.

Alain BOURDIN, La métropole des individus, Paris, Éditions de l'Aube, 2008.

Philippe CHAUDOIR (dir.), La ville évènementielle : temps de l'éphémère et espace festif, Géocarrefour, 2007/3, vol. 82.

Maria GRAVARI-BARBAS, Édith FAGNONI (dir.), Métropolisation et tourisme, comment le tourisme redessine Paris, Belin, 2013.

Éric LAMBAIN, Le Consommateur Planétaire, Éditions le Pommier, 2015

Christian LEFÈVRE, Nathalie ROSEAU, Tommaso VITALE (dir.), De la ville à la métropole, les défis de la gouvernance, L'œil d'or, collection Critique et Cités, 2013.

Jacques NOYER, Bruno RAOUL, Isabelle PAILLIART (dir.), Medias et Territoires, L'espace public entre communication et imaginaire territorial, Septentrion Presses Universitaires, Collection Information-Communication, 2013. Dominique PAGÈS (dir) :

- « La métropole parisienne, entre récits, paroles et échanges », Quaderni, n ${ }^{\circ} 73$, hiver 2010.

- « Les Imaginaires de la métropole créative », Hors Série des Cahiers de la Métropole, Mairie de Paris, avril 2014.

Dominique PAGÈS, Les Cahiers de la métropole,
«Les promesses de lieux pour la Métropole en devenir : pour quels lieux ? », revue Pratiques en santé mentale, janvier 2013.

Caroline STEEL, Hungry City, How food shapes our lives, Éditions Vintage, 2012 (réédition). 
$\mathrm{N} \cdot \mathrm{O} \cdot \mathrm{T} \cdot \mathrm{E} \cdot \mathrm{S}$

1. Pour aller plus loin, D. Pagès, « La fabrique symbolique du Grand Paris : du visible au tangible, une métropole à bras le corps », Carrefour du génie urbain, EIVP, 2015.

2. Nombre d'évènements se sont ainsi mis à l'heure métropolitaine, bouleversant la géographie administrative en reliant centre et périphérie, tel Le Festival de l'OH ou Futurs en Seine (évènement hybride qui met en scène une métropole innovante, créative, celle des cultures numériques) ou encore, Nuits Blanches et la Fête des jardins, qui, en se répétant d'année en année, cristallisent les attentions et les attentes, accompagnent les transformations métropolitaines.

3. Voir l'ouvrage collectif de référence de Violaine Appel, Cécile Bando, Hélène Boulanger, Gaëlle Crenn, Valérie Croissant, Bénédicte Toullec, La mise en culture des territoires, nouvelles formes de culture évènementielle et initiatives des collectivités locales, Presses Universitaires de Nancy, 2008.

4. D'autres évènements sont annoncés : ainsi en mai 2016 se tiendra un concours Ubiculteurs à la Cité des Sciences et de l'Industrie (après la Cité de la Mode et du Design en 2015) ; il s'adressera aux jeunes, élèves, collégiens, lycéens, étudiants, en association avec Naturparif. Il s'agira pour eux d'imaginer des potagers de classe répondant à une problématique précise.

5. Éclosion urbaine, festival organisé du 23 au 25 octobre par l'équipe du Ground Countrol et La Sauge. Makers, start ups, grandes entreprises, architectes, urbanistes et artistes sont invités à partager leurs expériences au fil de conférences, de débats, d'ateliers et à questionner ce modèle d'une métropole agri-urbaine en émergence.

6. C'est un collectif qui a pour principal objectif de rendre la ville comestible partout où cela sera possible.
Il fait partie du mouvement citoyen des Villes en transition qui interroge la résilience des territoires (www. transitionnetwork.org et http://villeentransition.net ).

7. Celle-ci est hébergée à la Gaîté Lyrique dans le cadre des projets Scintillo et Creatis (résidence d'entreprises culturelles, incubateur et plateforme consacrée à l'entrepreneuriat et à l'innovation dans les champs culturels).

8. Ce guide 'sur le chemin des Locavores invite aussi à soutenir de petites exploitations et de bonnes conditions de travail, à se reconnecter avec les saisons, à s'approvisionner et à produire ensemble, à jardiner et retrouver pleinement la maîtrise de son alimentation'. Éditions Eyrolles, 2010.

9. Éditions C. Bonneton, Collection Paris Utile, 2012. 10. Une micro-ferme maraîchère associative, un ferme 'innovante, véritable terrain d'expérimentations écologiques ' dont la rhétorique rappelle celle des utopies réalisées. Le style visuel du site est enfantin (dessins, éventail de couleurs pastels (bleu, blanc, vert, gris), évocation d'une planète rondement joviale où se juxtaposent harmonieusement monuments urbains, objets et produits de l'agriculture). Le site offre aussi un mur de portraits d'agriculteurs urbains au travail, bricolant, semant, bêchant, récoltant,...

11. Installé dans l'ancienne Gare Ornano (petite ceinture), ce lieu composite (de restauration, de recyclage, de culture : une petite ferme urbaine, un potager collectif et une prairie mellifère, un système d'aquaponie mais aussi deux systèmes de compostage) se veut un lieu d'échanges et de sociabilité autour des valeurs d'écologie alimentaire et de développement durable. L'esthétique vintage et nostalgique de son site évoque un lieu hospitalier à une nature urbaine esthétisée.

12. Voir l'exposition 360 adn Terre : www.bercyvillage.com/edito-360-adn-terre. 


\section{$R \cdot E ́ \cdot S \cdot U \cdot M \cdot E$}

Le 'Grand Paris qui mange', ce sont aussi des signes, des symboles, des récits qui participent à rendre perceptible un territoire en transition alimentaire, à rendre tangible la métropolisation agriurbaine, à sensibiliser les habitants et les visiteurs à ce nouveau modèle de ville dite nourricière, comestible, fertile mais aussi à leur donner la parole sur ces transformations métropolitaines... Cet article propose ainsi de penser ensemble les processus d'évènementialisation, de patrimonialisation, de mise en tourisme mais aussi de mise en intrigue qui fondent la fabrique d'une métropole durable et plus spécifiquement agri-urbaine.

'Greater Paris who eats' are also the signs, symbols, and stories which help us perceive a transition in the food habits of an area and an agri-urban metropolisation, and which help sensitise both inhabitants and visitors to a new urban model for a city said to be nourishing, edible and fertile, at the same time giving them a voice in such metropolitan transformations. This article proposes a collective reflection on the processes underpinning the creation of events, cultural heritage, tourism as well as the narratives, which are the basis of the creation of a sustainable metropolis, more specifically an agri-urban one. 
